\title{
Effects of Exenatide on both fat mass loss and cardiometabolic risk in obese subjects with type 2 diabetes mellitus
}

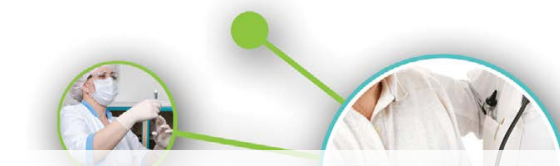

Objective: The regulation of glucose and adipose tissue metabolism by GLP1-receptor agonist is a possible treatment of T2D and obesity. These new pharmacologic agents target insulin resistance and metabolic disorders.

\begin{abstract}
Methods: Eighteen patients, 7 males and 11 females, were studied with T2D, divided into two groups: the first (I) was poorly controlled by metformin and sulfonylureas; the second (II) was treated only with metformin. Exenatide was administered at an initial dose of $5 \mu \mathrm{g}$ twice a day for one month, and then the dose was adjusted to $10 \mu \mathrm{g}$ twice a day throughout 24-26 weeks. From the beginning of therapy, each group was analyzed at time 0 and at 1 month (T1) and then 6 months (T2). At every time, anthropometric data, body composition by DXA and biochemical parameters were recorded.
\end{abstract}

Results: At baseline, all patients enrolled showed metabolic disorders and obesity. The comparison of both groups between baseline and T2 showed a significant decrease of body weight, total Fat Mass (FM) and HbA1c (\%), only in group (I) a statical reduction of Glycaemia and Total Cholesterol was observed. The Free Fat Mass (FFM) was never seen significantly different. The patients with $\mathrm{HbA1c}$ levels $>75 \mathrm{mmol} / \mathrm{mol}(9 \%)$ at baseline showed a greater decline of $\mathrm{HbA} 1 \mathrm{c}$ when compared to the patients with $\mathrm{HbA} 1 \mathrm{c}<75 \mathrm{mmol} / \mathrm{mol}$.

Conclusion: Exenatide improves glucose metabolism and total cholesterol levels. Moreover, Exenatide reduced total FM and did not affect FFM. Exenatide could be used to treat obesity in diabetic but also non-diabetic patients.

Summary: Exenatide, a GLP1-receptor agonist, is a pharmacological agent against obesity and type 2 diabetes mellitus. Exenatide decreases insulin resistance and lipids metabolism, and could be able to reduce fat mass and cardiovascular risk.

KEYWORDS: exenatide, GLP1-receptor agonist, diabetes mellitus, obesity, FM, FFM, cholesterol, HbA1c, DXA, cardiometabolic

\section{Introduction}

Obesity and glucose metabolism are tightly associated with a low degree chronic inflammation, related to pro-inflammatory cytokines produced by various cell types. These possibly regulate the adipose tissue metabolism $[1,2]$. GLP1-receptor agonists (RA) represent a relatively new class of pharmacologic agents for the treatment of T2D that reduce blood glucose by stimulating insulin secretion indirectly [3]. These drugs, by targeting insulin resistance, aim to prevent co-morbidities and possibly address chronic complications of the basic metabolic disorder. Additional to effects on blood glucose control, GLP1-RA, thanks to their pleiotropic actions, also induce delay of stomach emptying and, at level of hypothalamus, satiety $[3,10,11]$.

Previous studies proved GLP1-RA related body weight loss, coupled with fat tissue reshuffling, with no effects on the lean mass, hence on caloric expenditure [4-7]. Positive effects on blood hypertension and vascular protection have also been reported [8].

Because of the "diabesity" pandemics, introduction of drugs that act on basic factors that underlie pathogenesis of T2D and obesity, rather than only on glycemic control is welcome [9]. Few studies have so far addressed the effects of GLP1-RA on changes of body mass composition, and reduction of the fat mass, as assessed as by DXA, a main issue, in light of the role of adipose tissue on regulation of metabolism and cardiovascular risk.

Aim of the present study was to investigate the role of the GLP1-RA Exenatide on body composition, and in particular, on changes of the fat mass in obese patients (BMI $\geq 30 \mathrm{Kg} / \mathrm{m}^{2}$ ) with T2D and already on treatment with other antidiabetic agents.
Carmela Colica ${ }^{1}$, Sara Parrettini², Giuseppe Merra*3, Lorenzo Romano ${ }^{4}$, Laura Di Renzo ${ }^{5}$, Antonino De Lorenzo 5 \& Riccardo Calafiore ${ }^{2}$

${ }^{1}$ CNR, IBFM UOS of Germaneto, University "Magna Graecia" of Catanzaro, Campus "Salvatore Venuta", Germaneto, Catanzaro, Italy

${ }^{2}$ Department of Medicine, University of Perugia, Perugia, Italy

${ }^{3}$ Emergency Department, "A. Gemelli" General Hospital Foundation, Catholic University of Sacred Heart, Rome, Italy

${ }^{4}$ Specialisation School of Food Science, University of Rome "Tor Vergata", Rome, Italy

${ }^{5}$ Department of Biomedicine and Prevention, Section of Clinical Nutrition and Nutrigenomic, University of Rome "Tor Vergata", Rome, Italy

*Author for correspondence: giuseppe.merra@policlinicogemelli.it 


\section{Materials and methods}

T2D patients with $\mathrm{BMI} \geq 25 \mathrm{Kg} / \mathrm{m}^{2}$ and a disease history of at least 1 year, not fairly controlled by diet and Metformin, with or without sulfonylureas, for at least one month, were enrolled for study. All patients also were not on anti-hypertensive and blood fat lowering treatment, in absence of chronic micro-/macrovascular complications linked to diabetes.

Exenatide therapy was administered to all the recruited patients, at an initial dose of $5 \mu \mathrm{g}$ twice a day for one month to comply with GI tolerability; after this term, the drug dose was adjusted to $10 \mu \mathrm{g}$ twice daily throughout $24-26$ weeks of therapy.

All patients were evaluated at time 0 and subsequently at 1 month (T1) and 6 months (T2) from the beginning of the therapy.

At every check-up, the following parameters, weight $(\mathrm{Kg})$, height $(\mathrm{cm})$, waist (WC) and hip circumferences and waist/hips ratios $(\mathrm{W} / \mathrm{H})$ in $\mathrm{cm}$, were recorded. Body weight (BW) was evaluated, without clothing except underwear, to the nearest $0.1 \mathrm{~kg}$ on a calibrated scale (Invernizzi, Rome, Italy). Height $(\mathrm{H})$ was evaluated, without shoes, to the nearest $0.5 \mathrm{~cm}$ using a stadiometer (Invernizzi, Rome, Italy). BMI was obtained as BW $(\mathrm{kg})$ divided by square $\mathrm{H}(\mathrm{m} 2)$. Waist and hip circumferences were obtained with a plastic metric tape to the nearest $0.5 \mathrm{~cm}$, according to International Society for the Advancement of Kin anthropometry protocol and National Institute of Health Guidelines.

Fat Mass (FM) (kg), FM percental (\%) and Free Fat Mass (FFM) (kg) were measured using dual-energy X-ray absorptiometry (DXA; Model DPX, Lunar, Madison, WI, software revision 12.6).

DXA uses a constant potential X-ray source at $12.5 \mathrm{fJ}$ and a K-edge filter to achieve a congruent beam of stable dual-energy content (40 and 70 $\mathrm{keV})$. The coefficient of variation (CV) on this instrument for five subjects scanned six times over a nine months' period were $2.2 \%$ for fat mass and $1.1 \%$ for free fat mass (12). DXA calibration measurements were performed at the beginning of each examination and radiation exposure was $<8 \mu \mathrm{Sv}$. Biochemical parameters were assayed after a 12 hours fast, with special regard to fasting plasma glucose (FPG), total cholesterol (TC), triglycerides (TG) and highdensity cholesterol (HDL-C). Low density cholesterol (LDL-C) levels were assessed by the Friedewald formula. From these parameters cardiovascular risk indices (TOT CHOL/HDL; LDH/HDL; TG/HDL) were drawn. Glucose control was assessed by plasma HbA1c (\%) dosage by HPLC. By interview, subjects were asked to maintain their usual lifestyle habits and to report any illness or abnormality arising during the study.

\section{Statistics}

Statistics was analyzed by Software SPSS17.01. All variables were determined to verify normal distribution by the KolmogorovSmirnov test. Differences in the group at T0, T1 after one and half month, and T2 after 6 months of treatment with Exenatide were assessed by Student's paired T test. Group differences were evaluated by $T$ test for independent data. Data correlation was verified by Pearson coefficient determination. All data was expressed as mean $\pm S D$ and value range (Min-Max). Significance was assumed for $\mathrm{p}<0.05$.

\section{Results}

18 patients with T2D, 7 males and 11 females, were studied. All patients were under stable control therapy with oral agents. On the basis of the administered therapy regimens, they were divided into two groups: (I) $n=9$ included 3 males and 6 females with a history of T2D that was poorly controlled by dietary restrictions and treatment with metformin and sulfonylureas; (2) $n=9$, included 4 males and 5 females on treatment with diet and metformin only. TABLE 1 depicts basal parameters of enrolled patients.

\begin{tabular}{|c|c|c|c|c|}
\hline \multicolumn{2}{|c|}{ Table 1. Basal parameters of the enrolled patients. } \\
\hline & Mean \pm DS & Range & Mean \pm DS & Range \\
\hline Age $(\mathrm{yr})$ & $56.88 \pm 5.75$ & $49.00-66.00$ & $56.56 \pm 5.32$ & $47.00-64.00$ \\
\hline Weight $(\mathrm{Kg})$ & $92.85 \pm 21.53$ & $61.00-133.00$ & $100.94 \pm 16.79$ & $73.30-122.50$ \\
\hline BMI $\left(\mathrm{Kg} / \mathrm{m}^{2}\right)$ & $35.38 \pm 5.64$ & $28.04-44.16$ & $36.75 \pm 6.58$ & $28.81-47.45$ \\
\hline Waist $(\mathrm{cm})$ & $109.50 \pm 11.53$ & $90.00-124.00$ & $113.67 \pm 14.66$ & $90.50-130.00$ \\
\hline Hip (cm) & $116.25 \pm 14.98$ & $93.00-136.00$ & $118.94 \pm 15.26$ & $102.50-139.00$ \\
\hline W/H ratio & $0.95 \pm 0.07$ & $0.84-1.05$ & $0.94 \pm 0.07$ & $0.86-1.06$ \\
\hline FM (g) & $40003.78 \pm 13595.23$ & $21208.00-65115.00$ & $43981.78 \pm 11580.17$ & $27360.00-61972.00$ \\
\hline FFM (g) & $49666.56 \pm 10014.65$ & $37524.00-65974.00$ & $53766.56 \pm 9077.88$ & $38331.00-65752.00$ \\
\hline FM (\%) & $42.77 \pm 6.89$ & $29.20-48.70$ & $43.32 \pm 6.66$ & $30.20-52.40$ \\
\hline
\end{tabular}


Analysis of the anthropometric parameters and body composition, and comparison between them at different times, were associated with the following outcome. Group 1, as early as at 1 month of combined therapy (metformin + sulfonylurea + exenatide), showed decrease of body weight (T0 $92.85 \pm 21.53 \mathrm{Kg}$ vs. T1 $90.94 \pm 20.81 \mathrm{Kg}, \mathrm{p}<0.01)$ and Total FM (T0 $39711.19 \pm 13595.23 \mathrm{~g}$ vs. T1 $38656.89 \pm$ 12917.4 g, p<0.01). As far as FM was concerned, the decrease was statistically significant, close to $42.77 \pm 6.8 \%$ (T0) and $42.16 \pm 6.8 \%$ (T1), $<<0.05$. After 6 months of therapy (T2), statistically significant decline of body weight $(89.14 \pm 20.79 \mathrm{Kg}, \mathrm{p}<0.05)$, BMI $(33.92 \pm 5.2$ $\left.\mathrm{Kg} / \mathrm{m}^{2}, \mathrm{p}<0.05\right)$, FM $(37539.67 \pm 11505.10 \mathrm{~g}$, $\mathrm{p}<0.05)$ was observed. No significant variations of FFM ( $)$ ( $p>0.05)$ was apparent. In Group 1, a not statistically significant reduction of WC, $\mathrm{HC}$ and WHR was seen at T0 and T2. In Group 2, after a month of metformin + exenatide combined treatment, we observed significant decrease of body weight (T0 100.94 \pm 16.79 $\mathrm{Kg}$, T2 $93.03 \pm 14.59 \mathrm{Kg}, \mathrm{p}<0.05)$ and BMI (T0 $36.75 \pm 6.58 \mathrm{Kg} / \mathrm{m}^{2}$, T1 $35.73 \pm 6.70 \mathrm{Kg} /$ $\left.\mathrm{m}^{2}, \mathrm{p}<0.05, \mathrm{~T} 233.38 \pm 5.43 \mathrm{Kg} / \mathrm{m}^{2} \mathrm{p}<0.05\right)$. As for FM (g), between T0 $43981.78 \pm 11580.17$ $\mathrm{g}$ and T2 $36612.75 \pm 9744.03 \mathrm{~g}, \mathrm{p}<0.05$. As for FM, in percentage, we observed statistically significant decline between T0, T1 and T2 (T0 $43.32 \pm 6.66 \%$, T1 $41.56 \pm 6.78 \%, \mathrm{p}<0.01$, T2 $39.19 \pm 7.02 \%, \mathrm{p}<0.05)$. Also in group 2 no reduction of FFM (g) $(\mathrm{p}>0.05)$ was apparent.

As far as metabolic parameters were concerned, in both groups, reduction of either FPG (group 1: T0 $173.00 \pm 34.66 \mathrm{mg} / \mathrm{dl}$, T1 $164.67 \pm 37.80 \mathrm{mg} / \mathrm{dl}, \mathrm{T} 2152.89 \pm 21.54$ $\mathrm{mg} / \mathrm{dl}$; group 2: T0 $187.22 \pm 49.45 \mathrm{mg} / \mathrm{dl}, \mathrm{T} 1$ $145.33 \pm 30.45 \mathrm{mg} / \mathrm{dl}, \mathrm{T} 2145.38 \pm 25.731 \mathrm{mg} /$ dl) already significant at $\mathrm{T} 1$, or $\mathrm{HbA1c}$ (group 1: T0 $9.88 \pm 1.92 \%$. T2 $7.71 \pm 0.99 \%$ ) (Group 2: T0 $8.51 \pm 0.73 \%$. T2 $6.88 \pm 0.81 \%$ ) at T1 and T2, was shown (TABLE 2).

In terms of lipid parameters, no significant changes between the two groups, with the exception of decline of total plasma cholesterol in group $1 \quad(\mathrm{~T} 0=183.89 \pm 38.48 \mathrm{mg} / \mathrm{dl}$. $\mathrm{T} 1=164.33 \pm 32.25 \mathrm{mg} / \mathrm{dl} . \mathrm{T} 2=163.44 \pm 25.75$ $\mathrm{mg} / \mathrm{dl} . \mathrm{p}<0.05)$ were observed. We noticed improvement of blood pressure, both as far as systolic and diastolic values were concerned, in both groups (statistically significant statistics for the systolic values in the Metformin + exenatide combined treatment $(\mathrm{T} 0=142.89 \pm 11.60$ mmHg vs. T1 $132.11 \pm 11.45$ mmHg. $\mathrm{p}<0.05)$ (TABLE 3).

\begin{tabular}{|c|c|c|c|}
\hline \multirow{2}{*}{\multicolumn{4}{|c|}{$\begin{array}{r}\text { Table 2. Anthropometric and metabolic parameters } \\
\text { 1. Paired-test: }{ }^{1} p<0.05 ;{ }^{2} p<0.01 ;{ }^{3} p<0.001 . \text { WC }=\text { wa } \\
\text { WHR=waist-to-hip ratio; SBP=systolic blood pressur } \\
\text { Group } 1\end{array}$}} \\
\hline & & & \\
\hline & T0 & T1 & $\mathrm{T} 2$ \\
\hline Weight (Kg) & $92.85 \pm 21.53(61.00-133.00)$ & $90.94 \pm 20.81(61.50-130.70)^{2}$ & $89.14 \pm 20.79(63.80-132.00)^{1}$ \\
\hline $\mathrm{BMI}\left(\mathrm{Kg} / \mathrm{m}^{2}\right)$ & $35.38 \pm 5.64(28.04-44.16)$ & $34.66 \pm 5.55(26.93-43.17)$ & $33.92 \pm 5.20(27,68-43,60)^{1}$ \\
\hline WC $(\mathrm{cm})$ & $109.50 \pm 11.53(90.00-124.00)$ & $108.50 \pm 11.45(87.00-126.00)$ & $105.89 \pm 11.36(91.00-127.00)$ \\
\hline $\mathrm{HC}(\mathrm{cm})$ & $116.25 \pm 14.98(93.00-136.00)$ & $113.00 \pm 12.23(93.00-134.00)$ & $112.89 \pm 12.03(95.00-134.00)$ \\
\hline WHR & $0.95 \pm 0.07(0.84-1.05)$ & $0.96 \pm 0.07(0.85-1.04)$ & $0.94 \pm 0.07(0.84-1.06)$ \\
\hline Fat mass $(\mathrm{g})$ & $\begin{array}{c}39711.19 \pm 13595.23(21208.00- \\
65115.00)\end{array}$ & $\begin{array}{l}38656.89 \pm 12917.42 \\
(21546.00-62595.00)^{2}\end{array}$ & $\begin{array}{c}37539.67 \pm 11505.10 \\
(22760.00-61160)^{1}\end{array}$ \\
\hline $\begin{array}{l}\text { Free fat mass } \\
(\mathrm{g})\end{array}$ & $\begin{array}{c}49661.56 \pm 10014.65(37524.00- \\
65974.00)\end{array}$ & $\begin{array}{c}49138.78 \pm 9637.45(37998.00- \\
6371.00)\end{array}$ & $\begin{array}{l}49406.11 \pm 10066.84 \\
(38341.00-67717.00)\end{array}$ \\
\hline Fat mass (\%) & $42.77 \pm 6.89(29.20-48.70)$ & $42.16 \pm 6.81(29.10-48.30)^{1}$ & $41.52 \pm 5.46(30.70-46.30)$ \\
\hline $\begin{array}{c}\text { Glycemia (mg/ } \\
\text { dl) }\end{array}$ & $173.00 \pm 34.66(120.00-231.00)$ & $164.67 \pm 37.80(125.00-252.00)$ & $\begin{array}{c}152.89 \pm 21.54(125.00- \\
192.00)^{1}\end{array}$ \\
\hline $\mathrm{HbA1c} \%$ & $9.88 \pm 1.92(7.70-13.10)$ & $7.97 \pm 1.11(6.40-9.90)^{2}$ & $7.71 \pm 0.99(6.00-8.80) 1$ \\
\hline \begin{tabular}{|c|} 
Tot. cholesterol \\
$(\mathrm{mg} / \mathrm{dL})$
\end{tabular} & $183.89 \pm 38.48(139.00-257.00)$ & $\begin{array}{c}164.33 \pm 32.25(122.00- \\
223.00)^{1}\end{array}$ & $\begin{array}{c}163.44 \pm 25.75(131.00- \\
204.00)^{1}\end{array}$ \\
\hline $\mathrm{LDL}(\mathrm{mg} / \mathrm{dL})$ & $103.98 \pm 38.50(68.80-180.80)$ & $88.44 \pm 21.78(53.60-112.00)$ & $89.07 \pm 26.03(62.60-133.60)$ \\
\hline $\mathrm{HDL}(\mathrm{mg} / \mathrm{dL})$ & $44.44 \pm 19.72(25.00-94.00)$ & $43.78 \pm 7.19(37.00-61.00)$ & $42.11 \pm 9.14(30.00-62.00)$ \\
\hline $\mathrm{TG}(\mathrm{mg} / \mathrm{dL})$ & $177.33 \pm 85.66(90.00 .345 .00)$ & $160.56 \pm 89.74(78.00-360.00)$ & $161.33 \pm 71.98(92.00-283.00)$ \\
\hline $\mathrm{SBP}(\mathrm{mmHg})$ & $135.33 \pm 14.21(118.00-155.00)$ & $131.33 \pm 12.64(110.00-145.00)$ & $\begin{array}{c}129.22 \pm 13.94(112.00- \\
150.00)\end{array}$ \\
\hline $\mathrm{DBP}(\mathrm{mmHg})$ & $78.22 \pm 7.46(69.00-90.00)$ & $77.11 \pm 5.16(70.00-85.00)$ & $73.89 \pm 10.96(60.00-90.00)$ \\
\hline COL.TOT/HDL & $4.75 \pm 2.17(1.96-8.29)$ & $3.81 \pm 0.87(2.77-5.72)$ & $4.03 \pm 1.09(3.04-6.13)$ \\
\hline LDL/HDL & $2.82 \pm 1.79(0.77-5.83)$ & $2.05 \pm 0.57(1.25-2.87)$ & $2.24 \pm 0.98(1.46-4.11)$ \\
\hline TRIGL/HDL & $0.59 \pm 0.30(-0.02-0.88)$ & $0.52 \pm 0.22(0.25-0.97)$ & $0.56 \pm 0.20(0.31-0.83)$ \\
\hline
\end{tabular}


TABLE 4 compares the two study groups to outline eventual differences, with regard to percentage variations of the measured parameters at $\mathrm{T} 0 \mathrm{~T} 1$ and $\mathrm{T} 2$, and particularly to parameters that showed statistical changes throughout the study. Altogether, no differences, in terms of percent variations, between the two groups were observed.

\begin{tabular}{|c|c|c|c|}
\hline \multicolumn{4}{|c|}{ Group 2} \\
\hline & TO & $\mathrm{T} 1$ & $\mathrm{~T} 2$ \\
\hline Weight (Kg) & $100.94 \pm 16.79$ (73.30-122.50) & $97.98 \pm 15.86(72.10-117.10)$ & $\begin{array}{c}93.03 \pm 14.59(72.40- \\
109.70)^{1}\end{array}$ \\
\hline BMI $(\mathrm{Kg} / \mathrm{m} 2)$ & $36.75 \pm 6.58(28.81-47.45)$ & $35.73 \pm 6.70(28.10-47.21)^{1}$ & $33.38 \pm 5.43(26.50-41.86)$ \\
\hline WC $(\mathrm{cm})$ & $113.67 \pm 14.66(90.50-130.00)$ & $110.61 \pm 13.9988 .00-125.00)^{2}$ & $\begin{array}{l}106.88 \pm 13.90 \\
(86.00 .120 .00)\end{array}$ \\
\hline $\mathrm{HC}(\mathrm{cm})$ & $118.94 \pm 15.26(102.50-139.00)$ & $\begin{array}{c}114.39 \pm 12.24(102.00- \\
133.00)\end{array}$ & $\begin{array}{c}109.00 \pm 11.54(96.00- \\
129.00)^{1}\end{array}$ \\
\hline WHR & $0.94 \pm 0.07(0.86-1.06)$ & $0.95 \pm 0.09(0.86-1.09)$ & $0.95 \pm 0.07(0.88-1.03)$ \\
\hline Fat mass $(\mathrm{g})$ & $\begin{array}{l}43981.73 \pm 11580.17 \\
(27360.00-61972.00)\end{array}$ & $\begin{array}{l}41005.56 \pm 11327.39 \\
(24102.00-61331.00) 2\end{array}$ & $\begin{array}{l}36612.75 \pm 9744.0 \\
(19615.0-48769.0)\end{array}$ \\
\hline Free fat mass (g) & $\begin{array}{c}53766.56 \pm 9077.88(38331.00- \\
65752.00)\end{array}$ & $\begin{array}{l}53780.22 \pm 8330.56 \\
(40359.00-65752.00)\end{array}$ & $\begin{array}{l}53159.00 \pm 8293.80 \\
(42353.00-63958.00)\end{array}$ \\
\hline Fat mass (\%) & $43.32 \pm 6.66(30.20-52.40)$ & $41.56 \pm 6.78(28.90 .52 .40)^{2}$ & $39.19 \pm 7.02(24.90-47.20)^{1}$ \\
\hline Glycemia (mg/dL) & $187.22 \pm 49.45(127.00-265.00)$ & $\begin{array}{c}145.33 \pm 30.45(106.00- \\
200.00)^{1}\end{array}$ & $\begin{array}{c}145.38 \pm 25.731(107.00- \\
180.0)\end{array}$ \\
\hline $\mathrm{HbA1c} \%$ & $8.51 \pm 0.73(7.80-9.80)$ & $7.78 \pm 0.82(6.70-9.40)^{1}$ & $6.88 \pm 0.81(5.80-7.80)^{2}$ \\
\hline Tot.chol. (mg/dL) & $201.33 \pm 33.67(140.00-249.00)$ & $\begin{array}{c}194.44 \pm 44.67(112.00- \\
260.00)\end{array}$ & $\begin{array}{c}190.75 \pm 37.56(11.00- \\
234.00)\end{array}$ \\
\hline $\mathrm{LDL}(\mathrm{mg} / \mathrm{dL})$ & $126.73 \pm 34.62(71.80-191.20)$ & $120.12 \pm 32.32(65.20-168.20)$ & $\begin{array}{c}109.93 \pm 23.93(55.80- \\
133.20)\end{array}$ \\
\hline $\mathrm{HDL}(\mathrm{mg} / \mathrm{dL})$ & $43.44 \pm 12.27(30.00-63.00)$ & $43.67 \pm 12.05(28.00-65.00)$ & $49.25 \pm 13.27(29.00-67.00)$ \\
\hline $\mathrm{TG}(\mathrm{mg} / \mathrm{dL})$ & $155.78 \pm 23.20(137.00-207.00)$ & $153.27 \pm 49.44(94.00-269.00)$ & $\begin{array}{c}157.88 \pm 59.67(101.00- \\
299.00)\end{array}$ \\
\hline $\mathrm{SBP}(\mathrm{mmHg})$ & $142.89 \pm 11.60(120.00-160.00)$ & $\begin{array}{c}132.11 \pm 11.45(110.00- \\
145.00)^{1}\end{array}$ & $\begin{array}{c}129.88 \pm 11.36(113.00- \\
150.00)\end{array}$ \\
\hline $\mathrm{DBP}(\mathrm{mmHg})$ & $84.89 \pm 9.03(70.00-100.00)$ & $79.00 \pm 10.57(60.00-90.00)$ & $73.8 \pm 9.15(67.00-90.00)$ \\
\hline COL.TOT/HDL & $4.96 \pm 1.69(3.17-8.30)$ & $4.61 \pm 1.22(3.38-6.84)$ & $4.01 \pm 0.90(3.15-5.71)$ \\
\hline LDL/HDL & $3.19 \pm 1.51(1.74-6.37)$ & $2.87 \pm 0.94(1.91-4.43)$ & $2.31 \pm 0.60(1.72-3.25)$ \\
\hline TRIGL/HDL & $0.57 \pm 0.15(0.34-0.77)$ & $0.54 \pm 0.16(0.37-0.85)$ & $0.50 \pm 0.20(0.25-0.86)$ \\
\hline
\end{tabular}

\begin{tabular}{|c|c|c|c|c|c|c|c|c|}
\hline \multirow{3}{*}{$\Delta \%$} & \multicolumn{4}{|c|}{ Group 1} & \multicolumn{4}{|c|}{ Group 2} \\
\hline & \multicolumn{2}{|c|}{$\Delta \%(\mathrm{t} 0-\mathrm{t} 1)$} & \multicolumn{2}{|c|}{$\Delta \%(\mathrm{t} 0-\mathrm{t} 2)$} & \multicolumn{2}{|c|}{$\Delta \%(\mathrm{t} 0-\mathrm{t} 1)$} & \multicolumn{2}{|c|}{$\Delta \%(\mathrm{t} 0-\mathrm{t} 2)$} \\
\hline & Mean \pm DS & $\begin{array}{l}\text { Median } \\
\text { (Range) }\end{array}$ & $\begin{array}{c}\text { Mean } \pm \\
\text { DS }\end{array}$ & $\begin{array}{l}\text { Median } \\
\text { (Range) }\end{array}$ & $\begin{array}{l}\text { Mean } \pm \\
\text { DS }\end{array}$ & $\begin{array}{l}\text { Median } \\
\text { (Range) }\end{array}$ & $\begin{array}{l}\text { Mean } \pm \\
\text { DS }\end{array}$ & $\begin{array}{l}\text { Median } \\
\text { (Range) }\end{array}$ \\
\hline $\begin{array}{c}\Delta \% \\
\text { weight }\end{array}$ & $\begin{array}{c}-1.96 \pm \\
1.63\end{array}$ & $\begin{array}{c}-2.43 \\
(-4.09-0.82)\end{array}$ & $\begin{array}{l}-3.80 \pm \\
5.22\end{array}$ & $\begin{array}{c}-2.43 \\
(-12.08-4.49)\end{array}$ & $\begin{array}{c}-2.81 \pm \\
3.71\end{array}$ & $\begin{array}{c}-1.49 \\
(-10.45-0.09)\end{array}$ & \begin{tabular}{|c|}
$-5.56 \pm$ \\
5.75
\end{tabular} & $\begin{array}{c}-3.76 \\
(-15,59-0.09)\end{array}$ \\
\hline $\begin{array}{l}\Delta \% \\
\mathrm{BMI}\end{array}$ & $\begin{array}{c}-2.04 \pm \\
1.51\end{array}$ & $\begin{array}{c}-2.44 \\
(-4.10-0.82)\end{array}$ & $\begin{array}{c}-3.88 \pm \\
5.15\end{array}$ & $\begin{array}{c}-2.44 \\
(-12.08-4.56)\end{array}$ & $\begin{array}{c}-2.80 \pm \\
3.71\end{array}$ & $\begin{array}{c}-1.49 \\
(-10.44-0.10)\end{array}$ & $\begin{array}{c}-5.63 \pm \\
5.72\end{array}$ & $\begin{array}{c}-4.04 \\
(-15.59-0.10)\end{array}$ \\
\hline $\begin{array}{l}\Delta \% \\
F M\end{array}$ & $\begin{array}{c}-3.31 \pm \\
2.27\end{array}$ & $\begin{array}{c}-3.35 \\
(-6.08-1.59)\end{array}$ & $\begin{array}{c}-4.93 \pm \\
7.46\end{array}$ & $\begin{array}{c}-6.07 \\
(-14.34-7.32)\end{array}$ & $\begin{array}{c}-6.59 \pm \\
7.04\end{array}$ & $\begin{array}{c}-5.09 \\
(-22.77-0.00)\end{array}$ & $\begin{array}{c}-11.96 \pm \\
12.60\end{array}$ & $\begin{array}{c}-7.83 \\
(-33.81-1.82)\end{array}$ \\
\hline $\begin{array}{l}\Delta \% \\
\mathrm{FPG}\end{array}$ & $\begin{array}{l}-2.99 \pm \\
21.55\end{array}$ & $\begin{array}{c}0.78 \\
(-28.93- \\
41.67)\end{array}$ & $\begin{array}{c}-9.39 \pm \\
17.32\end{array}$ & $\begin{array}{c}-16.88 \\
(-26.67-24.17)\end{array}$ & $\begin{array}{c}-19.45 \pm \\
20.37\end{array}$ & $\begin{array}{c}-23.93 \\
(-39.90-22.05)\end{array}$ & $\begin{array}{c}-17.60 \pm \\
21.18\end{array}$ & $\begin{array}{c}-19.67 \\
(-43.19-25.98)\end{array}$ \\
\hline $\begin{array}{c}\Delta \% \\
H b A 1_{c}\end{array}$ & $\begin{array}{c}-17.94 \pm \\
12.31\end{array}$ & $\begin{array}{c}-17.82 \\
(-36,64-1)\end{array}$ & \begin{tabular}{|c|}
$-19.37 \pm$ \\
16.62
\end{tabular} & $\begin{array}{c}-16.67 \\
(-47.33-10.39)\end{array}$ & $\begin{array}{l}-8.42 \pm \\
8.22\end{array}$ & $\begin{array}{c}-7.59 \\
(-23.86-2.53)\end{array}$ & $\begin{array}{c}-18.98 \pm \\
14.52\end{array}$ & $\begin{array}{c}-17.39 \\
(-40.82-2.53)\end{array}$ \\
\hline $\begin{array}{c}\Delta \% \\
\text { TOT CHOL }\end{array}$ & $\begin{array}{c}-9.87 \pm \\
11.16\end{array}$ & $\begin{array}{c}-12.23 \\
(-22.22- \\
11.89)\end{array}$ & $\begin{array}{l}-9.61 \pm \\
12.67\end{array}$ & $\begin{array}{c}-13.25 \\
(-25.91-5.04)\end{array}$ & $\begin{array}{c}-3.97 \pm \\
11.90\end{array}$ & $\begin{array}{c}-2.53 \\
(-25.81-10.00)\end{array}$ & $\begin{array}{c}-2.55 \pm \\
12.64\end{array}$ & $\begin{array}{c}-3.38 \\
(-20.71-14.71)\end{array}$ \\
\hline
\end{tabular}




\begin{tabular}{|c|c|c|}
\hline & $\mathrm{Hb} 1 \mathrm{Ac}<75 \mathrm{mmol} / \mathrm{mol}$ & $\mathrm{Hb} 1 \mathrm{Ac}>75 \mathrm{mmol} / \mathrm{mol}$ \\
\hline$\Delta \%$ & \multicolumn{2}{|c|}{$\Delta \%(\mathrm{t} 0-\mathrm{t} 2)$} \\
\hline$\Delta \%$ Weight & $-4.39 \pm 4.85(-13.18--0.9)$ & $-4.89 \pm 6.25(-15.59-4.59)$ \\
\hline$\Delta \% \mathrm{BMI}$ & $-4.46 \pm 4.82(13.17--10)$ & $-4.97 \pm 6.17(-15.59-4.56)$ \\
\hline$\Delta \% \mathrm{FM}$ & $-6.59 \pm 9.48(-28.31-4.38)$ & $-10.08 \pm 11.92(-33.81-7.32)$ \\
\hline$\Delta \% \mathrm{HbA}_{\mathrm{c}}$ & $-10.52 \pm 10.77(-25.00-10.39)$ & $-29.34 \pm 13.42^{2}(-47.33-8.51)$ \\
\hline$\Delta \%$ Tot $\mathrm{CHOL}$ & $-1.61 \pm 8.97(-13.25-14.71)$ & $-15.17 \pm 10.52^{2}(-25.91-4.90)$ \\
\hline
\end{tabular}

Analyzing the variables correlations (percent variations of body weight, BMI and FM (\%) at T2), a positive correlation between percent variations of $\mathrm{HbAlc}$ and percent variation of FM (g) at T2 is evident.

Comparison of either anthropometric or metabolic parameters of the enrolled patients, with respect to initial $\mathrm{HbA1c}$ values (HbA1c cut off $=75 \mathrm{mmol} / \mathrm{mol}$ or $9 \%$ ), we have divided the patients in two cohorts. The assessment related to the period $\mathrm{T} 0-\mathrm{T} 2$, with evaluation of body weight, BMI, FM, HbAlc, and plasma total cholesterol (TABLE 5). In those patients showing HbA1c levels $>75 \mathrm{mmol} / \mathrm{mol}(9 \%)$, at T0, decline of HbA1c $(-29.34 \pm 13.42 \mathrm{mmol} /$ $\mathrm{mol}, \mathrm{p}<0.01)$ was greater and significant as compared to the group associated with HbA1c $<75 \mathrm{mmol} / \mathrm{mol}(-10.52 \pm 10.7 \mathrm{mmol} / \mathrm{mol})$. Total cholesterol also showed a statistically significant reduction in the HbAlc group $>75$ $\mathrm{mmol} / \mathrm{mol}$ compared to the group with $\mathrm{Hb} 1 \mathrm{Ac}$ $<75 \mathrm{mmol} / \mathrm{mol}(15.17 \pm 10.52 \mathrm{mg} / \mathrm{dl}(\mathrm{p}<0.01)$ vs. $-1.61 \pm 8.97 \mathrm{mg} / \mathrm{dl})$. However, BMI, body weight and FM did not differ between groups.

\section{Discussion}

We have examined the effects of Exenatide on reduction of FM and on metabolic profile of patients with T2D and observed that this shortacting GLP1-RA agent (Exenatide) induced both improvement of metabolic control and decline of adipose tissue.

T2D is based on insulin resistance at level of muscle and adipose tissue, in a contest of low-grade chronic systemic inflammation with progressive loss of $\beta$-cell function. However, other actors play a role, in the pathogenesis and progression of T2D, such as the GI tract (now thought to represent a real endocrine organ) that is associated with derangements of the enteroislet axis and decline of GLP1 production by the $\mathrm{L}$ cells $[9,10]$. Hence, treatment of DM cannot neglect addressing mechanisms that promote hyperglycemia, at level of both adipose tissue, and the incretins system [2,9]. GLP1RA are pleiotropic molecules, associated with subsequent glucose lowering, but also targeting additional organs. In fact, delay in stomach emptying coupled with induction of satiety at level of the NS both contribute on decrease of food intake [9]; peptides structurally related to GLP1 (exendin 4 and 9) induce lipolysis in the adipocytes of obese people [11]. Exenatide, a molecule similar to exendin 4, is a short-acting GLP1 analog, able to improve glycemic control (by reduction of both HbA1c and post-prandial plasma glucose) but also to lower body weight and ameliorate metabolic profile $[12,14]$. We have demonstrated, in the present study, decline of $\mathrm{HbA1c}$ at T2 with a $\Delta \%$ equal to -29.34 $\mathrm{mmol} / \mathrm{mol}$, that was statistically significant in all patients with starting levels of $\mathrm{HbA1}>>75$ $\mathrm{mmol} / \mathrm{mol}$, in accordance to previously published reports $[8,13]$.

As far as lipid profile is concerned, we observed improvement of total cholesterol and fractions plasma levels both, in patients treated with metformin + sulfonylhureas and in those on previous treatment with metformin only. While statistical significance was reaches only for total cholesterol plasma levels in Group 1, the observation time period was relatively short with low numbers of patients which could have conditioned missing statistics for other lipid parameters. In our study, exenatide seemed to reduce cardio-metabolic risk in both groups. Analisys of lipid profile is in agreement with a study of Drucker et al. in 2008 [8]. This report showed that $10 \mu \mathrm{g}$ of exenatide, administered twice daily, was associated with significant reduction of total plasma cholesterol. While many reports have focused, so far, on effects of incretins in improving metabolic control, a few studies have targeted the effects of these agents on body composition as assessed by DXA [14-17]. Garber et al. had shown that Liraglutide [15], a GLP1 analog, administered alone or in association with Metformin, resulted in significant reduction of percent FM, as compared to other drugs as glimepiride. Preservation of FFM was confirmed, in 2010, by Bunck et al. [14] on 69 patients with T2D on treatment with Metformin with insulin Glargine or Exenatide twice a day being added for one year. Results showed that one year of therapy with Exenatide reduced $\Delta \%$ of FM 
by $11 \%$, while no changes were observed in the patients treated with insulin Glargine. The study goes along with our own results where we observed a statistically significant decline of total FM by $4.93 \%(-2.172 \mathrm{Kg})$ in the first group treated with Exenatide + Metformin + sulfonylureas, and by $11.96 \%(-7.369 \mathrm{Kg})$ on the second group treated with Exenatide + Metformin. The lower weight and FM loss in Group 1 was likely associated with sulfonylureas addition, that often have been associated with body weight increase and risk and cardiovascular mortality raise $[17,18]$.

From our observations on decline of FM, we believe that Exenatide twice a day may represent a treatment option also in obese non-diabetic patients. This agrees with Bradley et al. [7], who showed that Exenatide, administered at a dose of $5 \mu \mathrm{g}$ twice a day for two weeks, and then $10 \mu \mathrm{g}$ twice a day, for the following 10 weeks, resulted in significant reduction of body weight, with decrease of FM by $1.3 \mathrm{Kg}$. No changes of FFM, or modifications of life style and dietary habits were applied. The study concluded that decline of body weight and FM related to reduction of calories intake, since no changes in caloric expenditure (TEF) was apparent. Finally, Chen et al. [16] documented decline of visceral adipose tissue (VAT) in 33 diabetic patients, on treatment with Exenatide for 18 weeks as an "add on" therapy to oral hypoglycemic agents. Decline of VAT in obese patients is associated with decrease of systemic chronic inflammation caused by pro-inflammatory cytokines, secreted by ectopic fat. Body weight decrease associated with selective FM loss, with no changes of FFM hence of basal metabolism, may permit access to management of pathogenic factors involved in the progressive deterioration of general metabolic and systemic conditions. Close correlation between glucose control and adipose tissue was proven by a positive correlation of percent variations between HbAlc and FM (g). In addition to DXA and biochemical analyzes, the evaluation of the effects of GLP1-RA, pre and post treatment, on fat tissue would require the characterization of fat deposits to deepen the metabolic state and the obesity phenotype. Recently, Magnetic Resonance (MR) was applied both in the study of changes of body fat distribution, and in the detection of brown adipose tissue with chemical shift-encoding based water fat MR imaging [19]. Therefore, MR could be used to analyze the effects of pharmacological and dietary treatments. Through this non-invasive technique, the pleiotropic effects of GLP1-RA on transformation and distribution of adipose tissue, obese phenotype and cardiovascular risk can be clarified. Free $\mathrm{f}$ diet, living habits and exercise was a limit of the study.

\section{Conclusion}

Preliminarily, Exenatide results in clear improvement of not only glucometabolic control of diabetic patients that did not respond, adequately, to treatment with oral agents alone or in combination, but also of total plasma cholesterol. This is likely associated with decline of cardiovascular risk. Moreover, exenatide unlike exogenous insulin does not affect FM [18] and is safer in terms of risk for hypoglycemia. It is possible to speculate that Exenatide would be a good candidate for treating obesity in diabetic but also non-diabetic patients, since its effect on declining body weight, selectively acting on FM (decline of VAT especially) and on preservation of FFM. Future studies will be needed to clarify the role of diet and exercise in relation to Exenatide treatment; in addition, the effects of GLP1-RA on the deposition of body fat should be clarified to understand the relationship between obesity phenotypes, metabolic disorders and cardiovascular risk. In relation to the results on the reduction of cardiovascular risk factors due to treatment with Exanetide, it will be necessary to evaluate the impact of GLP1-RA on coronary plaque regression or for the reduce of cardiovascular risk.

\section{Conflict of interest}

All the authors have no conflict of interest to declare. 


\section{References}

Park HS, Park JY, Yu R. Relationship of obesity and visceral adiposity with serum concentrations of CRP, TNFalpha and IL-6. Diabetes Res. Clin. Pract. 69(1), 29-35 (2005).

De Lorenzo A, Soldati L, Sarlo F, et al. New obesity classification criteria as a tool for bariatric surgery indication. World J. Gastroenterol. 22(2), 681-703 (2016).

Meier JJ. GLP-1 receptor agonists for individualized treatment of type 2 diabetes mellitus. Nat. Rev. Endocrinol. 8(12), 728-742 (2016).

Cuthbertson DJ, Irwin A, Gardner CJ, et al. Improved glycaemia correlates with liver fat reduction in obese, type 2 diabetes, patients given glucagon-like peptide-1 (GLP-1) receptor agonists. PLoS One.7 (12), e50117 (2012).

Astrup A, Carraro R, Finer N, et al. Safety, tolerability and sustained weight loss over 2 years with the once-daily human GLP-1 analog, liraglutide. Int. J. Obes. (Lond). 36(6), 843-854 (2012).

Samson SL, Sathyanarayana P, Jogi M, et al. Exenatide decreases hepatic fibroblast growth factor 21 resistance in nonalcoholic fatty liver disease in a mouse model of obesity and in a randomised controlled trial. Diabetologia. 54(12), 3093-3100 (2011).

Bradley DP, Kulstad R, Racine N, et al. Alterations in energy balance following exenatide administration. Appl. Physiol. Nutr. Metab. 37(5), 893-899 (2012).
Hong JY, Park KY, Kim BJ, et al. Effects of Short-Term Exenatide Treatment on Regional Fat Distribution, Glycated Hemoglobin Levels, and Aortic Pulse Wave Velocity of Obese Type 2 Diabetes Mellitus Patients. Endocrinol. Metab. (Seoul) 31(1), 80-85 (2012).

Defronzo RA. Banting Lecture. From the triumvirate to the ominous octet: a new paradigm for the treatment of type 2 diabetes mellitus. Diabetes. 58(4), 773-795 (2009).

Flint A, Raben A, Astrup A, Holst JJ. Glucagon-like peptide 1 promotes satiety and suppresses energy intake in humans. J. Clin. Invest. 101(3), 515520 (2009).

Sancho V, Trigo MV, Martín-Duce A, et al. Effect of GLP-1 on D-glucose transport, lipolysis and lipogenesis in adipocytes of obese subjects. Int. J. Mol. Med. 17(6), 1133-1137 (2006).

Di Renzo L, Bigioni M, Bottini FG, et al. Normal Weight Obese syndrome: role of single nucleotide polymorphism of IL-1 5Ralpha and MTHFR 677C-->T genes in the relationship between body composition and resting metabolic rate. Eur. Rev. Med. Pharmacol. Sci. 10(5), 235-245 (2006).

Drucker DJ, Buse JB, Taylor K, et al. Exenatide once weekly versus twice daily for the treatment of type 2 diabetes: a randomised, openlabel, non-inferiority study. Lancet. 372(9645), 1240-1250 (2008).

Bunck MC, Diamant M, Eliasson B, et al. Exenatide affects circulating cardiovascular risk biomarkers independently of changes in body composition. Diabetes Care. 33(8), 1734-1737 (2010).

Jendle J, Nauck MA, Matthews DR, et al. Weight loss with liraglutide, a once-daily human glucagon-like peptide- 1 analogue for type 2 diabetes treatment as monotherapy or added to metformin, is primarily as a resultof a reduction in fat tissue. Diabetes Obes. Metab. 11(12), 1163-1172 (2009).

Chen J, Sun H, Wang X, et al. Effects of exenatide on fat redistribution in centrally obese patients with type 2 diabetes mellitus. Int. J. Clin. Exp. Med. 10(2), 3470-3477 (2017).

Azimova K, San Juan Z, Mukherjee D. Cardiovascular safety profile of currently available diabetic drugs. Ochsner J. 14(4), 616-632 (2014).

Mäkimattila S, Nikkilä K, Yki-Järvinen H. Causes of weight gain during insulin therapy with and without metformin in patients with Type II diabetes mellitus. Diabetologia. 42(4), 406-412 (1999).

Franz D, Weidlich D, Freitag F, et al. Association of proton density fat fraction in adipose tissue with imaging-based and anthropometric obesity markers in adults. Int. J. Obes. (Lond). (2017). 